\section{Serviço fluvial de emergência: características e fatores relacionados à evolução clínica dos atendidos}

\author{
A riverine emergency health service: \\ characteristics and factors related to patients' \\ clinical evolution
}

\section{Servicio fluvial de emergencia: características y factores relacionados con la evolución clínica de los atendidos}

\section{Resumo}

O objetivo deste estudo foi descrever o perfil dos usuários e a assistência prestada pelo Serviço de Atendimento Móvel de Urgência (SAMU) fluvial em comunidades ribeirinhas amazônicas e identificar fatores relacionados à evolução dos pacientes durante o atendimento desse serviço. Trata-se de um estudo descritivo realizado em duas etapas: na primeira, os dados dos atendimentos dos indivíduos realizados pelo serviço de 2009 a 2015 foram a fonte de informações; na segunda, a coleta de dados foi prospectiva durante o primeiro semestre de 2016 e a evolução dos pacientes foi avaliada pelo Rapid Emergency Medicine Score. Em todos os períodos analisados, cerca de $90 \%$ dos atendimentos incluíram remoção, variando ao longo dos anos o tipo de embarcação mais utilizada no transporte. As causas dos atendimentos foram, predominantemente, sintomas e sinais de etiologia desconhecida ou causas externas. Dos pacientes transportados pelo serviço no primeiro semestre de 2016, 68,5\% mantiveram o quadro clinico, 18\% pioraram e 13,5\% melhoraram durante o atendimento. Para os atendidos nesse período, os tempos médios, resposta e total de atendimento fluvial foram 84 e 172 minutos, respectivamente. Houve associação entre evolução e ocorrências com serpentes venenosas, presença de dor aguda, idade, gravidade inicial e destino do usuário. Causas de atendimento tiveram relação com o agravamento durante a assistência e os que pioraram eram mais jovens, de menor gravidade e foram, com maior frequência, encaminhados para hospitais. O perfil dos usuários e dos atendimentos do SAMU fluvial apontou para necessidade de revisão dos propósitos e de estruturação desse serviço.

Emergências; Serviços Médicos de Emergência; Transportes; Evolução Clínica; Fatores de Risco

\author{
Ellen de Fátima Caetano Lança 1 \\ Lêda Lima Sobral 2 \\ Rita de Cássia Almeida Vieira 3 \\ Lilia de Souza Nogueira 3 \\ Regina Marcia Cardoso de Sousa 3
}

doi: 10.1590/0102-311X00281120

\author{
Correspondência \\ L. S. Nogueira \\ Escola de Enfermagem, Universidade de São Paulo. \\ Av. Dr. Enéas de Carvalho Aguiar 419, São Paulo, \\ SP 04660-000, Brasil. \\ lilianogueira@usp.br \\ 1 Escola de Enfermagem de Manaus, Universidade Federal do \\ Amazonas, Manaus, Brasil. \\ 2 Núcleo de Educação Permanente em Urgência, Serviço de \\ Atendimento Móvel de Urgência, Manaus, Brasil. \\ 3 Escola de Enfermagem, Universidade de São Paulo, São Paulo, \\ Brasil.
}




\section{Introdução}

O atendimento pré-hospitalar, presente em vários países, é considerado um importante serviço de atenção aos casos de urgência e emergência. No Brasil, o Serviço de Atendimento Móvel de Urgência (SAMU) é o principal componente móvel do atendimento pré-hospitalar. Este serviço é organizado conforme os protocolos do Ministério da Saúde de forma regionalizada e hierarquizada com o objetivo de prestar assistência às urgências e emergências precocemente, com pessoal treinado e equipamentos adequados, mantendo as condições de vida do paciente até a chegada em um serviço de saúde adequado para seu atendimento e prevenindo sequelas significativas 1,2 .

O SAMU brasileiro presta assistência padronizada e opera com uma central de regulação médica, acionada via telefone, que oferece à população duas modalidades de atendimento: a de Suporte Básico de Vida, realizada por unidade tripulada por pessoal de nível técnico para atender, com auxílio à distância dos médicos da central de regulação, casos sem risco imediato de vida; e outra de Suporte Avançado de Vida, que conta obrigatoriamente com um médico e um enfermeiro entre os tripulantes da unidade. Para cada solicitação de atendimento, os médicos reguladores designam a modalidade adequada que será enviada ao local perante as informações obtidas na chamada telefônica de acionamento do serviço 2,3 .

No Brasil, o SAMU utiliza, para atendimento, frotas terrestres (ambulâncias e motolâncias), aéreas (aeronaves) e fluviais (embarcações). Portanto, além das ambulâncias consideradas transporte terrestre convencional, há meios alternativos, como as aeronaves e "motolâncias", utilizadas em situações específicas para atendimentos em áreas remotas ou de tráfego intenso, e as "ambulanchas”, embarcações para atender os moradores de regiões ribeirinhas 2,3 .

No Amazonas, Manaus centraliza os recursos especializados de saúde no estado. A estrutura precária e deficiente dos serviços nos demais municípios tornou a rede de saúde da capital responsável pelo atendimento à população ${ }^{4}$. Além disso, a região amazônica possui características geográficas peculiares, distribuição populacional esparsa e sazonalidade das águas dos rios; consequentemente, as comunidades ribeirinhas têm dificuldade de acessar as unidades de saúde especializadas 5 .

Para minimizar essas dificuldades, muitas dessas comunidades ribeirinhas são atendidas no âmbito pré-hospitalar pelo SAMU fluvial, que teve início em 2006 na cidade de Manaus 6.

Ainda que haja propostas de serviços de atendimento pré-hospitalar com uso de embarcações para transporte hidroviário, no Brasil 2,3,7 e em outros países 8,9 , não foram encontradas na literatura informações mais detalhadas sobre esses serviços, como o perfil dos usuários e os fatores relacionados com a evolução dos pacientes durante o translado.

Estudos têm procurado identificar fatores relacionados com a evolução de pacientes atendidos no atendimento pré-hospitalar terrestre 10,11, considerando variáveis como tempos de atendimento, perfil sociodemográfico e clínico do usuário, procedimentos realizados pelo serviço e distância percorrida até o local de destino. A associação entre as características do atendimento pré-hospitalar terrestre e os resultados durante essa fase do atendimento é alvo de interesse para pesquisa tanto quando se consideram as frotas terrestres como quando se examinam os meios alternativos de transporte, como o fluvial, utilizados pelo atendimento pré-hospitalar. Diante de um cenário pouco conhecido do serviço fluvial, foi objetivo da atual pesquisa descrever o perfil dos usuários e a assistência prestada pelo SAMU fluvial em comunidades ribeirinhas amazônicas e identificar fatores relacionados à evolução dos pacientes durante o atendimento desse serviço.

Conhecer as características dos usuários e dos atendimentos do SAMU fluvial e as variáveis envolvidas na evolução dos atendidos pode auxiliar na tomada de decisão para a realização de procedimentos durante o atendimento e sobre o local de destino, além de direcionar o treinamento das equipes. Essas informações são também essenciais para o planejamento, a implementação e a avaliação de serviços móveis de emergência que utilizam embarcações para atendimento em locais de difícil acesso. 


\section{Métodos}

Estudo observacional, descritivo realizado em Manaus, um dos 62 municípios do Amazonas, com área de $11.401 .092 \mathrm{~km}^{2}$, situado ao norte do estado, cerca de $580 \mathrm{~km}$ do Estado do Pará e $690 \mathrm{~km}$ do Estado de Roraima, na junção dos rios Negro e Amazonas, os quais fazem parte da bacia amazônica (Instituto Brasileiro do Geografia e Estatística. Cidades e Estados. https://www.ibge.gov.br/cidades-e-estados, acessado em 19/Nov/2020). Um total de 57 comunidades ribeirinhas encontra-se na área de cobertura do SAMU fluvial deste município. Essas comunidades estão localizadas 100km acima de Manaus à margem esquerda do rio Negro (37 comunidades) e 100km abaixo, à margem esquerda do rio Amazonas (20 comunidades).

O estudo foi desenvolvido em duas etapas, sendo que os atendimentos realizados pelo SAMU fluvial de Manaus foram as unidades das análises. Na primeira etapa, a casuística incluiu todos os atendimentos registrados pelo SAMU fluvial no período de janeiro de 2009 a dezembro de 2015. A fonte de coleta de dados utilizados nesta etapa do estudo foi o banco de estatísticas do SAMU fluvial, no qual estão compiladas as informações dos atendimentos realizados a partir do ano de 2009. Desse banco de dados, obtiveram-se, retrospectivamente, as seguintes informações: mês e ano do atendimento, número de atendidos, faixa etária e sexo dos usuários, comunidade do atendimento, tipo de embarcação, natureza da ocorrência e número de pacientes removidos.

$\mathrm{Na}$ segunda etapa, foram coletados, prospectivamente, na base do SAMU fluvial e na central de regulação do serviço, os dados primários dos indivíduos com 15 anos ou mais atendidos pelo SAMU fluvial no período de janeiro a junho de 2016. A ficha clínica individual do atendimento pré-hospitalar prestado pelo SAMU fluvial e terrestre foram fontes de informação desta fase da pesquisa. A consulta à ficha do atendimento fluvial foi realizada no momento da chegada das embarcações na base do SAMU fluvial e a ficha de atendimento terrestre foi consultada na central de regulação. A hora do acionamento das embarcações foi obtida no banco de dados do rádio operador, profissional da equipe da central de regulação que controla a frota de veículos do SAMU.

Nesta segunda etapa, foram coletados os mesmos dados da primeira, além de procedimentos realizados no atendimento pré-hospitalar (conforme descrito na ficha do SAMU fluvial), tempos de atendimento, parâmetros fisiológicos no início e final do atendimento pré-hospitalar fluvial e local de destino do paciente.

O Rapid Emergency Medicine Score (REMS), instrumento de medida utilizado neste estudo para identificar a evolução dos usuários do atendimento pré-hospitalar fluvial, foi elaborado para predizer mortalidade hospitalar em pacientes adultos admitidos em serviço de emergência 12 . Este índice foi definido por meio de análise de regressão logística em uma casuística de pacientes com idade mínima de 15 anos, sendo essa a idade estabelecida na segunda etapa do atual estudo.

A localização da comunidade ribeirinha (rio Negro ou Amazonas) e a distância percorrida pelas embarcações do local de atendimento até a base fluvial em Manaus foram estabelecidas pela identificação da comunidade que ocorreu o atendimento. A natureza da ocorrência foi descrita conforme capítulos da Classificação Internacional de Doenças e Problemas Relacionados à Saúde - 10a Revisão (CID-10. https://apps.who.int/iris/handle/10665/42980, acessado em 27/Abr/2020). Nas análises de associação relacionadas à natureza das ocorrências, foram consideradas, além dos capítulos, as categorias da CID-10.

Os tempos de atendimento foram analisados conforme segue: - tempo de resposta: minutos consumidos pelo SAMU fluvial entre o acionamento da embarcação para o atendimento até a chegada à comunidade da ocorrência; - tempo de cena: minutos entre a chegada à comunidade da ocorrência até a saída; - tempo de transporte fluvial: minutos entre a saída da comunidade da ocorrência até a chegada à base do SAMU em Manaus; - tempo total do atendimento pré-hospitalar fluvial: minutos desde o acionamento da embarcação até a chegada do paciente à base do SAMU em Manaus; - tempo total do atendimento pré-hospitalar: minutos desde o acionamento da embarcação para o atendimento até a chegada do paciente ao serviço de saúde de destino (incluiu o tempo de atendimento pré-hospitalar terrestre).

Para definir a gravidade e a evolução dos pacientes atendidos, foi utilizado o REMS. Esta escala foi desenvolvida para serviços de emergência e tem sido indicada como um bom instrumento para predizer mortalidade hospitalar. O REMS é calculado pela somatória da pontuação atribuída à idade e 
às variáveis fisiológicas: pressão arterial média, frequência cardíaca, frequência respiratória, Escala de Coma de Glasgow e saturação periférica de oxigênio. A pontuação mínima do REMS é zero e a máxima, 26 pontos, sendo que as maiores pontuações indicam maior gravidade 13 . Pesquisadores descreveram a pontuação do REMS $<6$ como baixo risco de morrer; pontuação $\geq 6$ e $\leq 13$ como risco intermediário; e $>13$ pontos indicativo de alto risco de evoluir a óbito 13 .

A variável dependente desta investigação - evolução da condição fisiológica no atendimento pré-hospitalar fluvial - foi identificada pela diferença da pontuação do REMS entre o atendimento inicial e final do SAMU fluvial. Resultados da subtração (REMS inicial - REMS final) inferiores a zero indicaram evolução para piora clínica do paciente durante o atendimento desse serviço. Valores dessa subtração iguais a zero ou positivos indicaram, respectivamente, manutenção ou melhora do quadro clínico do paciente durante o atendimento pré-hospitalar fluvial. O valor numérico da diferença do REMS (inicial - final) foi considerado variável dependente em análises de correlação de Spearman quando a idade, o escore do REMS inicial e as variáveis relacionadas aos tempos de atendimento pré-hospitalar (tempo resposta, cena, de transporte fluvial, total fluvial e até a chegada ao serviço de saúde de destino) foram analisados.

A fim de traçar o perfil demográfico e clínico dos pacientes atendidos pelo SAMU fluvial nas hidrovias do Amazonas e do atendimento a eles prestado, foi realizada análise descritiva dos dados, em frequências absolutas e relativas. Nos casos de variáveis numéricas, foram calculadas medidas de tendência central para completar a caracterização dos pacientes e do serviço.

Para verificar se houve diferença entre os pacientes atendidos pelo SAMU fluvial que pioraram ou não durante o atendimento, utilizaram-se os testes qui-quadrado e exato de Fisher nas análises das variáveis categóricas. O teste qui-quadrado foi aplicado para verificar associação entre a piora durante o atendimento pré-hospitalar e sexo. O teste exato de Fisher foi utilizado para as demais variáveis categóricas, visto que, nesses casos, os pressupostos para a aplicação do teste qui-quadrado não foram atendidos. Para os tempos de atendimento pré-hospitalar (variáveis numéricas), foi aplicado o teste não paramétrico de Mann-Whitney, além do coeficiente de correlação de Spearman, alternativas não paramétricas, uma vez que os pressupostos para testes paramétricos não foram atendidos. O software R (http://www.r-project.org) foi utilizado para a realização das análises descritivas e inferenciais, e o nível de significância adotado foi de $5 \%$.

O projeto deste estudo foi aprovado pelo Comitê de Ética em Pesquisa da Escola de Enfermagem, Universidade de São Paulo (processo no 1.400.141) e teve autorização da Comissão de Ética e Pesquisa da Secretaria Municipal de Saúde de Manaus. Houve dispensa da aplicação do Termo de Consentimento Livre e Esclarecido.

\section{Resultados}

Nos anos de 2009 a 2015, o SAMU fluvial de Manaus realizou 2.002 atendimentos. O maior número foi efetuado no ano de 2010, seguido de 2012, com média de uma ocorrência diária. A partir de 2013, houve, anualmente, redução do número de atendimentos (Tabela 1). A distribuição dos atendimentos realizados nos anos de 2009 a 2015, de acordo com os meses de sua ocorrência, não evidenciou convergência entre os meses do ano que ocorreu maior ou menor número de atendimentos, assim como entre as estações do ano. A frequência de atendimentos mensais diferiu bastante a cada ano, variando de 20 (fevereiro) a 36 (julho), em 2009; 21 (junho) a 40 (outubro), em 2010; 13 (dezembro) a 41 (agosto), em 2011; 2 (dezembro) a 48 (junho), em 2012; 13 (outubro) a 25 (abril), em 2013; 12 (abril) a 22 (dezembro), em 2014; e 7 (dezembro) a 25 (julho), em 2015.

Na Tabela 1, observa-se que a frequência de homens atendidos pelo SAMU fluvial foi ligeiramente superior nos anos de 2010 e 2015, e próxima à observada em mulheres nos demais anos. Na distribuição segundo a faixa etária, a maioria dos usuários tinha menos de 35 anos e quase sempre houve declínio na frequência dos atendidos com o aumento da idade. Os menores de 15 anos foram os mais frequentes em 2012 e tiveram expressiva frequência nos demais anos.

Do total de 2.002 atendimentos, 72,9\% pertenciam às comunidades distribuídas ao longo do rio Negro e 27,1\%, do rio Amazonas. Na Tabela 1, pode-se observar que o número de atendimentos nesses sete anos foi sempre superior em comunidades localizadas no rio Negro. Em relação à distância das 
Atendimentos realizados pelo Serviço de Atendimento Móvel de Urgência (SAMU) fluvial, segundo ano, considerando as características dos usuários e dos atendimentos. Manaus, Amazonas, Brasil, 2009-2015.

\begin{tabular}{|c|c|c|c|c|c|c|c|}
\hline $\begin{array}{l}\text { Características } \\
\text { dos usuários e } \\
\text { atendimentos }\end{array}$ & $\begin{array}{c}2009 \\
{[n=342]} \\
\%\end{array}$ & $\begin{array}{c}2010 \\
{[n=371]} \\
\%\end{array}$ & $\begin{array}{c}2011 \\
{[\mathrm{n}=342]} \\
\%\end{array}$ & $\begin{array}{c}2012 \\
{[n=350]} \\
\%\end{array}$ & $\begin{array}{c}2013 \\
{[n=212]} \\
\%\end{array}$ & $\begin{array}{c}2014 \\
{[\mathrm{n}=202]} \\
\%\end{array}$ & $\begin{array}{c}2015 \\
{[n=183} \\
\%\end{array}$ \\
\hline \multicolumn{8}{|l|}{ Sexo } \\
\hline Feminino & 52,0 & 42,9 & 46,8 & 46,9 & 54,2 & 49,0 & 43,7 \\
\hline Masculino & 48,0 & 57,1 & 53,2 & 53,1 & 45,8 & 51,0 & 56,3 \\
\hline \multicolumn{8}{|l|}{ Faixa etária (anos) } \\
\hline$<15$ & 25,1 & 27,2 & 26,6 & 32,0 & 22,1 & 19,3 & 18,0 \\
\hline$\geq 15 \mathrm{e}<35$ & 36,9 & 34,8 & 36,3 & 29,7 & 32,6 & 38,1 & 34,4 \\
\hline$\geq 35 \mathrm{e}<55$ & 19,0 & 20,8 & 19,9 & 18,0 & 19,4 & 19,8 & 25,7 \\
\hline$\geq 55$ e $<75$ & 14,3 & 13,7 & 14,3 & 16,9 & 18,4 & 14,4 & 14,2 \\
\hline$\geq 75$ & 4,7 & 3,5 & 2,9 & 3,4 & 7,5 & 8,4 & 7,7 \\
\hline \multicolumn{8}{|l|}{ Localização da } \\
\hline Rio Amazonas & 36,3 & 24,5 & 19,3 & 19,4 & 34,4 & 40,6 & 20,8 \\
\hline Rio Negro & 63,7 & 75,5 & 80,7 & 80,6 & 65,6 & 59,4 & 79,2 \\
\hline \multicolumn{8}{|l|}{ Distância $(\mathrm{km})$ * } \\
\hline$\leq 25$ & 38,6 & 56,6 & 59,6 & 62,6 & 36,8 & 30,2 & 34,4 \\
\hline$>25$ e $\leq 50$ & 17,5 & 16,2 & 12,6 & 11,1 & 21,2 & 21,8 & 21,9 \\
\hline$>50$ e $\leq 75$ & 14,9 & 10,5 & 7,6 & 6,0 & 11,8 & 15,8 & 22,4 \\
\hline$>75$ e $\leq 100$ & 29,0 & 16,7 & 20,2 & 20,3 & 30,2 & 32,2 & 21,3 \\
\hline \multicolumn{8}{|l|}{ Remoção ** } \\
\hline Sim & 98,8 & 87,6 & 89,8 & 90,6 & 92,0 & 94,6 & 90,2 \\
\hline Não & 1,2 & 12,4 & 10,2 & 9,4 & 8,0 & 5,4 & 9,8 \\
\hline
\end{tabular}

* Distância percorrida pelas embarcações da base fluvial em Manaus até a comunidade ribeirinha do atendimento;

** Atendimento com remoção dos pacientes do SAMU fluvial.

comunidades até a base fluvial em Manaus, foram mais frequentes os atendimentos às comunidades mais próximas da base fluvial. Em todo o período analisado, uma ampla maioria dos atendidos pelo SAMU fluvial foi removida para Manaus (87,6\% a 98,8\%).

O tipo de embarcação mais utilizada para a remoção dos usuários nos anos de 2009 e 2010 foi a Unidade de Suporte Avançado Fluvial (Tabela 2). Nos quatro anos subsequentes, a Unidade Suporte Básico Fluvial passou a ser mais usada e, em 2015, a embarcação de Suporte Avançado Fluvial, com ajustes em sua tripulação, voltou a ser a mais usual.

Na Figura 1, pode-se observar que, nos anos de 2009 a 2012, as ocorrências de maior incidência foram as relacionadas ao capítulo XX (Causas externas de morbidade e mortalidade) da CID-10. De 2013 a 2015, as ocorrências relacionadas ao capítulo XVIII (Sintomas, sinais e achados anormais) apresentaram o maior percentual. Ocorrências inseridas nos capítulos IX (Doenças do aparelho circulatório), X (Doenças do aparelho respiratório), XI (Doenças do aparelho digestivo) e XV (Gravidez e puerpério) tiveram uma frequência expressiva em quase todos os anos do período.

No período de janeiro a junho de 2016, o SAMU fluvial realizou 119 atendimentos. Destes, 26 $(21,8 \%)$ foram para menores de 15 anos e 93 para aqueles com idade igual ou superior, sendo estes últimos incluídos na segunda etapa deste estudo. Para esses 93 atendimentos, foram registrados 145 procedimentos (média de 1,5 procedimento/atendimento). O procedimento mais frequente foi o acesso venoso periférico, instalado em quase todos os atendidos (97,8\%), seguido por reposição volêmica (17,2\%). Os curativos, assim como a oxigenoterapia, foram realizados em 11,8\% da amostra; 


\section{Tabela 2}

Atendimentos com remoção pelo Serviço de Atendimento Móvel de Urgência (SAMU) fluvial, segundo modalidade da unidade fluvial. Manaus, Amazonas, Brasil, 2009-2015. Manaus, Amazonas, Brasil, 2009-2015.

\begin{tabular}{lcc}
\hline Ano & $\begin{array}{c}\text { Unidade de Suporte Avançado Fluvial } \\
\mathbf{n}(\%)\end{array}$ & $\begin{array}{c}\text { Unidade de Suporte Básico Fluvial } \\
\mathbf{n}(\%)\end{array}$ \\
\hline 2009 & $255(75,4)$ & $83(24,6)$ \\
2010 & $235(72,3)$ & $90(27,7)$ \\
2011 & $129(42,0)$ & $178(58,0)$ \\
2012 & $31(9,8)$ & $286(90,2)$ \\
2013 & $37(19,0)$ & $158(81,0)$ \\
2014 & - & $191(100,0)$ \\
2015 & $118(72,0)$ & $47(28,0)$ \\
\hline
\end{tabular}

\section{Figura 1}

Distribuição dos atendimentos realizados pelo Serviço de Atendimento Móvel de Urgência (SAMU) fluvial, segundo o ano e a natureza das ocorrências. Manaus, Amazonas, Brasil, 2009-2015.

6 Lança EFC et al.

Tabela 2

Atendimentos com remoção pelo Serviço de Atendimento Móvel de Urgência (SAMU) fluvial, segundo modalidade da unidade fluvial. Manaus, Amazonas, Brasil, 2009-2015. Manaus, Amazonas, Brasil, 2009-2015.

\begin{tabular}{ccc}
\hline Ano & $\begin{array}{c}\text { Unidade de Suporte Avançado Fluvial } \\
\mathbf{n}(\%)\end{array}$ & $\begin{array}{c}\text { Unidade de Suporte Básico Fluvial } \\
\mathbf{n}(\%)\end{array}$ \\
\hline 2009 & $255(75,4)$ & $83(24,6)$ \\
2010 & $235(72,3)$ & $90(27,7)$ \\
2011 & $129(42,0)$ & $178(58,0)$ \\
2012 & $31(9,8)$ & $286(90,2)$ \\
2013 & $37(19,0)$ & $158(81,0)$ \\
2014 & - & $191(100,0)$
\end{tabular}

\footnotetext{
* Inclui os capítulos I (Algumas doenças infecciosas e parasitárias), II (Neoplasias - tumores), III (Doenças do sangue e dos órgãos hematopoiéticos e alguns transtornos imunitários), IV (Doenças endócrinas, nutricionais e metabólicas), V (Transtornos mentais e comportamentais), VI (Doenças do sistema nervoso), VII (Doenças do olho e anexos), VIII (Doenças do ouvido e da apófise mastoide), XII (Doenças da pele e do tecido subcutâneo), XIII (Doenças do sistema osteomuscular e do tecido conjuntivo) e XIV (Doenças do aparelho geniturinário) da Classificação Internacional de Doenças e Problemas Relacionados à Saúde - 10ạ Revisão (https://apps.who.int/iris/handle/10665/42980, acessado em 27/Abr/2020).
} 
imobilizações e administração de medicamentos foram procedimentos utilizados em 6,5\% e 5,4\% dos casos, respectivamente. Dois $(2,2 \%)$ atendidos foram submetidos à ressuscitação cardiopulmonar e houve um caso $(1,1 \%)$ em que foi feita hemostasia por compressão direta do ferimento.

Na Tabela 3, são descritas as estatísticas dos tempos mensurados no atendimento pré-hospitalar fluvial dos 89 atendidos que foram removidos nesta etapa do estudo $(95,7 \%)$. Houve grande variabilidade dos tempos de atendimento pré-hospitalar, conforme se pode observar nos valores do desviopadrão das médias e amplitude entre valores máximos e mínimos.

O percentual dos pacientes removidos pelo SAMU fluvial e que foram encaminhados para hospital foi de $44,9 \%$ (40 pacientes); dentre eles, sete foram para hospital especializado em doenças tropicais e infecciosas. O serviço de saúde de pronto atendimento recebeu $37,1 \%(n=33)$ dos removidos; para maternidade, foram 15 casos $(16,9 \%)$ e $1(1,1 \%)$ usuário com transtornos mentais foi encaminhado para o ambulatório psiquiátrico.

A média do REMS inicial foi de 2,7 e do REMS final de 2,9 pontos. Desvio padrão de 3,6, valor mínimo de zero e valor máximo de 23 pontos foram observados tanto para o REMS inicial quanto para o final do atendimento pré-hospitalar fluvial. Valores do REMS inicial $\geq 6 \mathrm{e} \leq 13$ foram observados em 17,9\% dos atendidos e maior que $13 \mathrm{em}$ um único paciente. Quanto às mudanças do REMS entre o início e o final do atendimento, a maioria dos usuários $(\mathrm{n}=61 ; 68,5 \%)$ atendidos e transportados pelo SAMU fluvial de Manaus teve diferença da pontuação do REMS inicial e final igual a zero, isto é, mantiveram o quadro clínico durante o atendimento fluvial; 16 pacientes (18\%) tiveram resultado da subtração negativo, ou seja, apresentaram piora do quadro clínico durante o atendimento, e 12 pacientes $(13,5 \%)$ apresentaram melhora (diferença positiva) durante o atendimento pré-hospitalar fluvial.

O resultado do teste qui-quadrado indicou que os usuários que pioraram não diferiram dos demais em relação ao sexo. Não existiu uma relação de dependência entre a piora ou não do paciente e as seguintes características dos atendimentos: rio e distância em que se localizava a comunidade ribeirinha, tipo de embarcação no transporte, modalidade de atendimento terrestre e procedimentos que foram realizados durante o atendimento pré-hospitalar fluvial. Os tempos de atendimento também não estiveram associados com a evolução dos usuários, segundo o teste não paramétrico de Mann-Whitney e o coeficiente de correlação de Spearman.

A natureza das ocorrências que motivaram o atendimento não se associou com a piora dos pacientes atendidos pelo SAMU fluvial quando classificadas nos capítulos que compõem a CID-10. Entretanto, conforme se observa na Tabela 4, na análise das categorias desses capítulos, duas delas foram associadas à evolução do paciente no atendimento pré-hospitalar fluvial: contato com serpentes venenosas (valor de $\mathrm{p}=0,039$ ) e dor aguda (valor de $\mathrm{p}=0,005$ ).

Tabela 3

Estatísticas descritivas dos tempos em minutos dos atendimentos com remoção $(n=89)$ realizados pelo Serviço de Atendimento Móvel de Urgências (SAMU) fluvial. Manaus, Amazonas, Brasil, janeiro a junho de 2016.

\begin{tabular}{lccccc}
\hline $\begin{array}{l}\text { Estatísticas } \\
\text { descritivas }\end{array}$ & Resposta & Cena & $\begin{array}{c}\text { Transporte } \\
\text { fluvial }\end{array}$ & $\begin{array}{c}\text { Tempo (minutos) } \\
\text { Total do } \\
\text { atendimento pré- } \\
\text {-hospitalar fluvial }\end{array}$ & $\begin{array}{c}\text { Total do atendimento } \\
\text { pré-hospitalar }\end{array}$ \\
\hline Média & 84 & 18 & 70 & 172 & 198 \\
Mediana & 70 & 15 & 60 & 140 & 173 \\
Desvio padrão & 48,3 & 14,0 & 40,1 & 87,5 & 86,6 \\
Amplitude & 261 & 95 & 175 & 405 & 400 \\
Mínimo & 14 & 5 & 10 & 45 & 65 \\
Máximo & 275 & 100 & 185 & 450 & 465 \\
\hline
\end{tabular}


Aspectos relacionados à piora dos pacientes removidos pelo Serviço de Atendimento Móvel de Urgências (SAMU) fluvial, em casos de contato com serpentes venenosas, dor aguda e tipo de serviço de saúde de destino. Manaus, Amazonas, Brasil, janeiro a junho de 2016.

\begin{tabular}{|c|c|c|c|}
\hline & \multicolumn{2}{|c|}{ Piora } & \multirow[t]{3}{*}{ Valor de $p$ * } \\
\hline & $\operatorname{Sim}[n=16]$ & Não [n = 73] & \\
\hline & n (\%) & n (\%) & \\
\hline \multicolumn{4}{|l|}{ Contato com serpentes venenosas } \\
\hline Sim & $3(18,8)$ & $2(2,7)$ & 0,039 \\
\hline Não & $13(81,2)$ & $71(97,3)$ & \\
\hline \multicolumn{4}{|l|}{ Dor aguda } \\
\hline Sim & $3(18,8)$ & - & 0,005 \\
\hline Não & $13(81,2)$ & $73(100,0)$ & \\
\hline \multicolumn{4}{|l|}{ Serviço de saúde de destino } \\
\hline Serviço de pronto atendimento & $3(18,8)$ & $30(41,1)$ & \\
\hline Maternidade & $1(6,3)$ & $14(19,2)$ & 0,037 \\
\hline Hospital & $12(74,9)$ & $28(38,4)$ & \\
\hline
\end{tabular}

* Teste exato de Fisher.

Observa-se, também na Tabela 4, que existiu uma relação de dependência entre a piora do paciente e o serviço de saúde de destino (valor de $\mathrm{p}=0,037$ ) e que a maioria dos pacientes que tiveram piora foi encaminhada para serviços hospitalares $(74,9 \%)$.

Os resultados da aplicação do coeficiente de correlação de Spearman mostraram que a idade e o REMS inicial tiveram associação com mudança do REMS no atendimento pré-hospitalar fluvial (valor de $\mathrm{p}=0,043$ e 0,030, respectivamente). Os coeficientes de correlação foram $+0,215$ para idade e $+0,192$ para o REMS inicial.

\section{Discussão}

A análise das atividades do SAMU fluvial de Manaus nos anos de 2009 a 2015 mostrou abrupta redução dos atendimentos após o ano de 2012, chegando, em 2015, a uma diminuição de 50\% em relação ao ano de 2010. Embora haja na região períodos de chuvas e de mudanças nos níveis dos rios, não houve evidências de sazonalidade nos atendimentos conforme esperado, visto que as enchentes favorecem os surtos de doenças, principalmente as transmitidas por vetores, como malária e dengue, bem como as doenças de veiculação hídrica, como leptospirose, hepatite A e doenças diarreicas. Nessa época, há também o aumento dos agravos relacionados a animais peçonhentos que são típicos das enchentes dos rios amazônicos 14 .

Acredita-se que alguns fatores tenham contribuído para redução dos atendimentos no transcorrer do período analisado: o fortalecimento de outros serviços de atendimento à saúde das comunidades ribeirinhas, a inauguração da Ponte Rio Negro, em outubro de 2011, que melhorou a comunicação terrestre entre algumas comunidades e a capital, e diminuiu a frota fluvial descentralizada.

Até o ano de 2011, duas “ambulanchas”, Unidades de Suporte Básico Fluvial, eram distribuídas no rio Negro e duas no rio Amazonas, com o objetivo de atender a demanda das comunidades ribeirinhas e diminuir o tempo de resposta para moradores das localidades mais distantes. A partir desse ano, o SAMU fluvial passou a contar com uma embarcação básica descentralizada em cada rio e, em 2014, esse número diminuiu para uma unidade locada no rio Amazonas. Essa redução foi resultado das dificuldades de manter a tripulação das embarcações de locais distantes da capital. 
A maioria dos atendidos pelo SAMU fluvial foi transportada para serviços de saúde em Manaus e houve grande variabilidade no tipo de embarcação utilizada no transporte durante os anos em que os dados foram analisados; no entanto, no ano de 2015 e primeiro semestre de 2016, embora a Unidade de Suporte Avançado Fluvial tenha sido a embarcação que predominou, a tripulação dessas embarcações foi composta somente por enfermeiros, técnicos de enfermagem e arrais.

A falta de médicos nas ambulâncias e de fixação desses profissionais no SAMU é um problema recorrente em diversos estados brasileiros, sendo a Região Norte uma das mais atingidas no país 3 . Vale lembrar que um tema bastante discutido nos últimos tempos é a implantação de atendimento pré-hospitalar com unidades de Suporte Intermediário de Vida, cuja equipe é formada por um enfermeiro, um técnico ou auxiliar de enfermagem e um motorista, similar ao observado nas embarcações de Manaus a partir de 2015.

Outra questão estrutural envolvida na variabilidade no tipo de embarcação utilizada é a manutenção e renovação da frota do SAMU. No ano de 2014, as Unidades de Suporte Avançado Fluvial de Manaus estiveram em manutenção, razão pela qual não foram observados atendimentos dessa modalidade (Tabela 2). Há uma grave dificuldade de provimento rápido de manutenção de ambulâncias, apontada por coordenadores estaduais de urgência e especialistas em diferentes regiões do país; além disso, a renovação da frota tem sido mais lenta do que seu desgaste ${ }^{3}$.

Essas condições estruturais relacionadas ao quantitativo de profissionais e embarcações disponíveis reduzem a frota e tornam a existência dos recursos, além da sua necessidade, os critérios fundamentais para determinar a unidade encaminhada para uma ocorrência 3 .

Os atendimentos pré-hospitalares fluviais foram mais frequentes nas comunidades próximas da base fluvial e localizadas no rio Negro, visto que esse rio tem maior número de comunidades ribeirinhas quando comparado ao rio Amazonas, e grande parte delas está concentrada próxima a Manaus. Além disso, o rio Amazonas é uma importante via de ligação entre Manaus e outras grandes cidades da Região Norte, como Santarém e Belém, no Estado do Pará. Por isso, há maior número de alternativas de transporte para Manaus no rio Amazonas em relação ao rio Negro, e essa maior facilidade de transporte provavelmente contribuiu para a menor frequência de atendimentos do SAMU fluvial nesse rio.

Ainda que os atendimentos em comunidades ribeirinhas mais próximas tenham sido os mais frequentes, foram expressivas as ocorrências em locais mais distantes e elevados os tempos de atendimento pré-hospitalar fluvial. Ao estudar esses tempos, observou-se que o tempo médio de resposta para os pacientes removidos foi muito acima do tempo ideal de até 8 minutos 15,16,17 e do tempo resposta apontado em cidades brasileiras, que variou de 2 a 39 minutos 6,15,18.

No presente estudo, o tempo médio de cena foi de 18 minutos, corroborando com a média de 10 a 20 minutos encontrada em outros estudos 6,15. A média de tempo de transporte fluvial foi de 70 minutos, discrepando da média de tempo estimado no transporte terrestre, entre 5 e 30 minutos 6 . Houve grande variabilidade desses tempos, provavelmente relacionada à distância e às condições de acesso das comunidades ribeirinhas, que dependem da sazonalidade das águas da região, da correnteza dos rios, do fluxo de outras embarcações e até mesmo do acúmulo de lixo no percurso.

Ainda que o tempo de cena tenha sido condizente com outros serviços de atendimento pré-hospitalar, os tempos de resposta e de transporte fluvial prolongado, inerentes ao atendimento em localidades distantes e de difícil acesso, resultaram em longa duração dos tempos de atendimento pré-hospitalar fluvial e total. O tempo mínimo, médio e máximo de resposta tem sido um dos indicadores estatísticos que avaliam o SAMU 2 , sendo considerado o principal indicador de qualidade do serviço ${ }^{3}$. Em geral, pouco é comentado sobre a importância desse tempo em pacientes de áreas de difícil acesso; no entanto há necessidade de considerar o contexto da organização dos serviços para avaliar esse tempo, uma vez que eles dependem de uma série de fatores, como diferenças geográficas, tamanho da frota, localização de ambulâncias, entre outros.

Por outro lado, o excessivo tempo de resposta traz questionamentos sobre a compatibilidade do transporte fluvial para casos de urgências em saúde em locais de difícil acesso, e os serviços aeromédicos, vitais para grandes distâncias, são alternativas para transporte de pacientes. Esses serviços são utilizados na região amazônica e custeados pelos estados 3 . Contudo há de se pesar, nesse contexto, que a hidroviária é a principal via de transporte na região que permite comunicação entre Manaus e os demais municípios e comunidades ribeirinhas. 
O SAMU fluvial de Manaus atende pessoas com diferentes tipos de agravos à saúde; no entanto o atendimento a vítimas de causas externas se destacou entre os demais, sendo motivo da assistência de 20,8\% a 34,8\% dos usuários do serviço. Estudos nacionais têm destacado a elevada frequência das causas externas entre as ocorrências atendidas pelo SAMU. Menores percentuais são observados nas ocorrências obstétricas e psiquiátricas 1,19,20.

Sendo o atendimento pré-hospitalar uma assistência temporária até que o paciente seja admitido em uma instituição para o tratamento definitivo, torna-se fácil compreender o destaque dos sintomas, sinais e achados anormais no SAMU. Esse capítulo da CID-10 refere-se a condições clínicas não tão bem definidas, uma vez que o necessário estudo do caso para se estabelecer um diagnóstico final ainda não foi realizado. Além disso, os casos incluídos nesse capítulo necessitam ser encaminhados a outros locais para investigação ou tratamento antes que o diagnóstico seja feito.

O estudo mostrou média de 1,5 procedimento por atendimento, sendo o acesso venoso periférico instalado em quase todos os usuários $(97,8 \%)$. A frequência desse procedimento foi elevada perante os $30,1 \%$ e 34,4\% realizados em atendimentos de serviços terrestres 18 . Provavelmente, a manutenção de uma via de acesso venoso foi um procedimento realizado pelo SAMU fluvial como medida de precaução, além de ser a via utilizada para reposição volêmica e administração de medicamentos em 17,2\% e 5,4\% dos casos, respectivamente. A longa permanência dos atendidos na embarcação e a ausência de diagnóstico e recursos para tratamento definitivo no atendimento pré-hospitalar tornaram o acesso venoso uma medida de segurança que permitiu, quando necessário, a intervenção medicamentosa imediata nos casos de agravamento do quadro clínico. Em consequência, a punção venosa superou expressivamente a execução de manobras e intervenções de manutenção de vida, previstas nos serviços de urgência.

Em geral, os dados relacionados com a pontuação do REMS indicaram que os usuários do SAMU fluvial apresentaram baixo risco de vida durante o atendimento fluvial (REMS médio inicial de 2,7 e final de 2,9 pontos). Por conseguinte a reduzida frequência de intervenções realizadas pelo SAMU fluvial, exceto punção venosa, e a gravidade leve dos usuários apontaram para inapropriado uso do serviço. Deficiências nos sistemas de saúde resultam em atendimento de casos em que não há urgência em serviços móveis de emergência atendendo às demandas de transporte de pacientes. Isso ocorre em serviços que utilizam diferentes tipos de transporte e é um fenômeno internacional 3.

A concentração de serviços médicos de média e alta complexidade em Manaus e a dispersão de parte da população do estado do Amazonas em um grande território fazem que, com frequência, os moradores do estado necessitem se locomover para Manaus para realizar exames e consultas com especialistas 4,5. Essa é uma forte demanda social na região que pode ser mais expressiva do que a necessidade do atendimento às emergências 3 e que deve ser considerada pelos gestores de saúde da região.

Nesse sentido, vale comentar que a análise da rede regional de saúde na região amazônica apontou grande investimento de recursos humanos e materiais nos atendimentos de urgências na perspectiva de contribuir para integração dos serviços de saúde regional; no entanto, a Rede de Urgência e Emergência não apresentou uma resposta efetiva para essa integração, principalmente em relação à atenção primária de saúde 4 .

O contato com serpentes venenosas e dor aguda foram causas de atendimento do atendimento pré-hospitalar fluvial que tiveram relação com o agravamento do paciente (diferença entre REMS inicial e REMS final inferior a zero). Os acidentes ofídicos e escorpiônicos são considerados agravos tropicais que acometem principalmente populações de baixa renda e que habitam em áreas rurais. No Brasil, em 2019, foram registrados 187.210 casos e 321 óbitos. Apesar de serem mais frequentes os casos de acidentes escorpiônicos no país, principalmente na Região Nordeste, o ofidismo é responsável por parcela expressiva dos óbitos (33,8\%) por animais peçonhentos, sendo a região Norte responsável pela maioria das notificações de acidentes por serpentes. No ano de 2019, foram notificados 3.071 acidentes por animais peçonhentos no Amazonas, dentre os quais 2.024 (65,9\%) foram provocados por serpentes, ocorrências que se destacaram por sua magnitude e gravidade (Departamento de Informática do SUS. http://tabnet.datasus.gov.br/cgi/deftohtm.exe?sinannet/ cnv/animaisAM.def, acessado em 10/Mar/2020).

A dor aguda é uma das principais queixas de entrada em serviços de emergência, e pesquisas mostraram frequências superiores dessa queixa em relação ao presente estudo 21,22 . A ocorrência de dor 
aguda tem sido associada a alterações nos sinais vitais, dependência em pacientes com dor lombar e parada cardiorrespiratória em pacientes com dor torácica 23,24.

Ainda em relação aos fatores associados ao agravamento do usuário do SAMU fluvial durante o atendimento, houve relação de dependência entre piora e encaminhamento para serviços hospitalares. Indubitavelmente, o agravamento da condição clínica durante a assistência do SAMU fluvial foi um indicador utilizado pelos profissionais do atendimento pré-hospitalar para encaminhamento às unidades hospitalares, visto que os parâmetros do REMS indicavam gravidade leve na maioria dos usuários (cerca de 80\%), no início e final do atendimento.

A idade e a pontuação inicial do REMS tiveram correlação significativa e positiva com a evolução desse escore durante o atendimento do SAMU fluvial. Sendo assim, quanto maior a idade e o REMS inicial, mais positiva foi a diferença entre REMS inicial e final, ou seja, os usuários mais velhos e mais graves, segundo REMS inicial, tenderam à melhora durante o atendimento do SAMU fluvial. A idade avançada e a condição clínica mais grave foram, provavelmente, sinais de alerta para os profissionais e resultaram em intervenções precoces e melhores desenlaces dos atendimentos durante o transporte fluvial.

Entre as limitações desta investigação, vale salientar que os menores de 15 anos, não incluídos nas análises da segunda etapa da pesquisa, foram importante parcela dos usuários do SAMU fluvial. Além disso, o seguimento dos atendidos pelo SAMU restringiu-se ao atendimento pré-hospitalar, não sendo realizadas análises sobre os desfechos após esse atendimento.

\section{Conclusão}

No geral, os resultados mostraram peculiaridades do SAMU fluvial de Manaus que necessitam ser consideradas no planejamento, implementação e avaliação do atendimento pré-hospitalar fluvial, tais como os longos percursos e a consequente demora do acesso aos pacientes, baixa gravidade na maioria dos usuários e longa permanência dos atendidos na embarcação. Esses resultados, juntamente com a variabilidade do tipo de embarcação utilizada nos atendimentos durante o período analisado e a baixa frequência de procedimentos de manutenção de vida realizados durante o transporte, trazem questionamentos sobre a compatibilidade do transporte fluvial para casos de urgências em saúde em locais de difícil acesso, a respeito do uso apropriado do serviço e sobre a estrutura para manutenção do atendimento.

No entanto a piora dos pacientes durante o atendimento não se associou com tempos de atendimento, distâncias percorridas e tipo de embarcação utilizada. A dor aguda e aos acidentes ofídicos se relacionaram com a piora do paciente no atendimento pré-hospitalar fluvial enquanto a idade mais avançada e a maior gravidade inicial, indicada pelo REMS, se associaram com a melhora durante o atendimento desse serviço. A piora também esteve associada com o destino dos pacientes, sendo, nesses casos, mais frequente o encaminhamento dos atendidos aos serviços hospitalares. 


\section{Colaboradores}

E. F. C. Lança e R. M. C. Sousa contribuíram na concepção do projeto, análise e interpretação dos dados; redação do artigo e revisão crítica relevante do conteúdo intelectual; aprovação final da versão a ser publicada; e são responsáveis por todos os aspectos do trabalho na garantia da exatidão e integridade de qualquer parte da obra. L. L. Sobral, R. C. A. Vieira e L. S. Nogueira contribuíram na redação do artigo, revisão crítica relevante do conteúdo intelectual; aprovação final da versão a ser publicada; e são responsáveis por todos os aspectos do trabalho na garantia da exatidão e integridade de qualquer parte da obra.

\section{Informações adicionais}

ORCID: Ellen de Fátima Caetano Lança (00000001-6014-2279); Lêda Lima Sobral (0000-00020061-4219); Rita de Cássia Almeida Vieira (00000001-9210-4445); Lilia de Souza Nogueira (00000001-5387-3807); Regina Marcia Cardoso de Sousa (0000-0002-2575-7937).

\section{Agradecimentos}

À Coordenação de Aperfeiçoamento de Pessoal de Nível Superior (CAPES, código 001) pelo financiamento.

\section{Referências}

1. Almeida PMV, Dell'Acqua MCQ, Cyrino CMS, Juliani CMCM, Palhares VC, Pavelqueires S. Analysis of services provided by SAMU 192: Mobile component of the urgency and emergency care network. Esc Anna Nery Rev Enferm 2016; 20:289-95.

2. Ministério da Saúde. Portaria no 1.010 , de 21 de maio de 2012. Redefine as diretrizes para a implantação do Serviço de Atendimento Móvel de Urgência (SAMU192) e sua Central de Regulação das Urgências, componente da Rede de Atenção às Urgências. Diário Oficial da União 2012; 22 mai.

3. O'Dwyer G, Konder MT, Reciputti LP, Macedo C, Lopes MGM. O processo de implantação do Serviço de Atendimento Móvel de Urgência no Brasil: estratégias de ação e dimensões estruturais. Cad Saúde Pública 2017; 33:e00043716.

4. Sousa ABL, Garnelo L, Mota PHS, Bousquat A. Regional health network in the Amazonian context: the case in Manaus, Entorno and Alto Rio Negro. Rev Bras Saúde Mater Infant 2017; 17 Suppl 1:S249-58.

5. Garnelo G, Sousa ABL, Silva CO. Health regionalization in Amazonas: progress and challenges. Ciênc Saúde Colet 2017; 22:1225-34.

6. Minayo MCS, Deslandes SF. Análise da implantação do sistema de atendimento pré-hospitalar móvel em cinco capitais brasileiras. Cad Saúde Pública 2008; 24:1877-86.

7. Siqueira SC, Jesus VSD, Oliveira Muniz JPD, Oliveira MSD, Santos ADA, Dos Santos ELB, et al. Operation of mobile emergency units: quilombolas' perception. J Nurs Health Sci 2016; 2:10-5.

8. Erasmus E, Robertson C, van Hoving DJ. The epidemiology of operations performed by the National Sea Rescue Institute of South Africa over a 5-year period. Int Marit Health 2018; 69:1-7.

9. Franklin RC, Peden AE, Brander RW, Leggat PA. Who rescues who? Understanding aquatic rescues in Australia using coronial data and a survey. Aust N Z J Public Health 2019; 43:477-83.

10. Christensen EF, Larsen TM, Jensen FB, Bendtsen MD, Hansen PA, Johnsen SP, et al. Diagnosis and mortality in prehospital emergency patients transported to hospital: a populationbased and registry-based cohort study. BMJ Open 2016; 6:e011558.

11. Yu W, Chen H, Lv Y, Deng Q, Kang P, Zhang L. Comparison of influencing factors on outcomes of single and multiple road traffic injuries: a regional study in Shanghai, China (2011-2014). PLoS One 2017; 12:e0176907.

12. Olsson T, Terent A, Lind L. Rapid Emergency Medicine Score: a new prognostic tool for in-hospital mortality in nonsurgical emergency department patients. J Intern Med 2004 255:579-87. 
13. Bulut M, Cebicci H, Sigirli D, Sak A, Durmus O, Top AA, et al. The comparison of modified early warning score with rapid emergency medicine score: a prospective multicentre observational cohort study on medical and surgical patients presenting to emergency department. Emerg Med J 2014; 31:476-81.

14. Souza RF, Nascimento SL. Diseases and aggravations in the context of the largest gradual floods in the state of Amazonas - Brasil. Hygeia 2017; 13:139-47.

15. Cabral ELS, Castro WRS, Florentino DRM, Viana DA, Costa Junior JF, Souza RP, et al. Response time in the emergency services. Systematic review. Acta Cir Bras 2018; 33:1110-21.

16. Mell HK, Mumma SN, Hiestand B, Carr BG, Holland T, Stopyra J. Emergency medical services response times in rural, suburban, and urban areas. JAMA Surg 2017; 152:983-4.

17. Wang HE, Mann NC, Jacobson KE, Ms MD, Mears G, Smyrski K, et al. National characteristics of emergency medical services responses in the United States. Prehosp Emerg Care 2013; 17:8-14.

18. Hora RS, Paiva ED, Sampaio ES, Oliveira JA, Souza VRS, Brandão PC. Characterization of the urgency mobile service (Serviço de Atendimento Móvel de Urgência, SAMU) for clinical emergencies. REME 2019; 23:e1265.
19. Dias JMC, Lima MSM, Dantas RAN, Costa IKF, Leite JEL, Dantas DV. Profile of state prehospital mobile emergency care service. Cogitare Enferm 2016; 21:1-8

20. Battisti GR, Branco A, Caregnato RCA, Oliveira MMC. Profile of service and satisfaction of users of the Mobile Emergency Care Service (SAMU). Rev Gaúcha Enferm 2019; 40:e20180431.

21. Mura P, Serra E, Marinangeli F, Patti S, Musu M, Piras I, et al. Prospective study on prevalence, intensity, type, and therapy of acute pain in a second-level urban emergency department. J Pain Res 2017; 10:2781-8.

22. Rodrigues ISA, Oliveira LMM, Fernandes FECV, Teles MEV. Prevalence of acute pain in patients attending the emergency room. Rev Dor 2017; 18:327-31.

23. Grotle M, Foster NE, Dunn KM, Croft P. Are prognostic indicators for poor outcome different for acute and chronic low back pain consulters in primary care? Pain 2010; 151:790-7.

24. Inamasu J, Miyatake $S$, Tomioka $H$, Shirai $T$, Ishiyama M, Komagamine J, et al. Prognostic significance of acute pain preceding out-ofhospital cardiac arrest. Emerg Med J 2011; 28:613-7. 


\section{Abstract}

The study aimed to describe the profile of patients and the care provided by the Mobile Riverine Emergency Medical Service (SAMU) in river-dwelling communities of the Amazon and to identify factors related to patients' evolution during care by this service. This descriptive study was conducted in two stages: in the first, the data were obtained on care for individuals in the service from 2009 to 2015; in the second, the data collection was prospective during the first semester of 2016, and patients' evolution was assessed with the Rapid Emergency Medicine Score. In all the periods analyzed, some $90 \%$ of the care included medical evacuation, varying over the years as to the type of boat used most frequently in transporting the patients. The most frequent reasons for care were signs and symptoms of unknown etiology and external causes. Of the patients transported by the service in the first semester of 2016, 68.5\% maintained their clinical status, $18 \%$ worsened, and $13.5 \%$ improved during care. For patients seen during this period, the mean response and total riverine care times were 84 and 172 minutes, respectively. There was an association between evolution and incidents involving venomous snakes, acute pain, age, initial severity, and destination of the patient. Causes of care were related to worsening clinical status during care, and patients who worsened were younger and in less serious condition and were more frequently evacuated to hospitals. The profile of patients and care in the riverine SAMU pointed to the need for a revision of this service's objectives and structuring.

Emergencies; Emergency Medical Services; Transportation; Clinical Evolution; Risk Factors

\section{Resumen}

El objetivo de este estudio fue describir el perfil de los usuarios y la asistencia prestada por el Servicio de Atención Móvil de Urgencia (SAMU) fluvial en comunidades ribereñas amazónicas, así como identificar factores relacionados con la evolución de los pacientes durante la atención de ese servicio. Se trata de un estudio descriptivo realizado en dos etapas: en la primera, la fuente de información fueron los datos de las consultas de los individuos, realizadas por el servicio de 2009 a 2015; en la segunda, la recogida de datos fue prospectiva durante el primer semestre de 2016 y la evolución de los pacientes fue evaluada por el Rapid Emergency Medicine Score. En todos los periodos analizados, cerca de un $90 \%$ de las consultas incluyeron desplazamiento, variando a lo largo de los años el tipo de embarcación más utilizada en el transporte. Las causas de la atención fueron, predominantemente, sintomas y señales de etiología desconocida o causas externas. De los pacientes transportados por el servicio en el primer semestre de 2016, un $68,5 \%$ mantuvieron el cuadro clínico, $18 \%$ empeoraron y 13,5\% mejoraron durante la atención. Para los atendidos en ese periodo, los tiempos medios, respuesta y total de atención fluvial, fueron $84 y$ 172 minutos, respectivamente. Hubo asociación entre evolución y ocurrencias con serpientes venenosas, presencia de dolor agudo, edad, gravedad inicial y destino del usuario. Las causas de atención estuvieron relacionadas con el agravamiento durante la asistencia, y quienes empeoraron eran más jóvenes, de menor gravedad y fueron, con mayor frecuencia, dirigidos a hospitales. El perfil de los usuarios y de la atención del SAMU fluvial apuntó la necesidad de una revisión de los propósitos, así como de la estructuración de ese servicio.

Urgencias Médicas; Servicios Médicos de Urgencia; Transportes; Evolución Clínica; Factores de Riesgo
Recebido em 25/Set/2020

Versão final apresentada em 21/Nov/2020

Aprovado em 04/Jan/2021 